\title{
Germanica
}

\section{„Ein Schritt zurück in die exquisiteste Barbarei“ - Mit Deutschland in der Südsee: Christian Krachts metahistoriographischer Abenteuerroman Imperium}

"A Step Back to the Most Exquisite Barbarity" - A German Trip to the South Seas: Christian Krachts's Metahistoriographical Adventure Novel Imperium "Le retour à la barbarie exquise»-Voyage allemand vers le Pacifique :

Imperium de Christian Kracht

\section{Robin Hauenstein}

\section{OpenEdition Journals}

Édition électronique

URL : http://journals.openedition.org/germanica/2658

DOI : 10.4000/germanica.2658

ISSN : 2107-0784

Éditeur

Université de Lille

Édition imprimée

Date de publication : 30 décembre 2014

Pagination : 29-45

ISBN : 9782913857346

ISSN : 0984-2632

Référence électronique

Robin Hauenstein, « "Ein Schritt zurück in die exquisiteste Barbarei“ - Mit Deutschland in der Südsee: Christian Krachts metahistoriographischer Abenteuerroman Imperium », Germanica [Online], 55 | 2014, Online erschienen am: 30 Dezember 2016, abgerufen am 06 Oktober 2020. URL : http:// journals.openedition.org/germanica/2658; DOI : https://doi.org/10.4000/germanica.2658 


\title{
„Ein Schritt zurück in die exquisiteste Barbarei" \\ - Mit Deutschland in der Südsee:
}

\author{
Christian Krachts metahistoriographischer \\ Abenteuerroman Imperium ${ }^{1}$
}

\author{
Robin HAUENSTEIN \\ Universität Wien
}

Christian Krachts neuester Roman ist eines der letzten Beispiele für eine Tendenz in der deutschen Literatur, die sich verstärkt für globalere Themen und weiter entfernte Weltregionen interessiert ${ }^{2}$. Den Beginn dieser Radius-Ausweitung markierten ab den 1970er Jahren die verschriftlichten persönlichen Reiseerlebnisse in Ländern der Dritten Welt, die der deutschsprachigen Literatur erstmals eine postkoloniale Perspektive sicherten. Zu den wesentlichen Aspekten dieser Perspektive gehört das narrative Eingestehen von Unsicherheit, von Irritationen und möglichen Irrtümern sowie der Begrenztheit des Erfahrungshorizonts, das so den olympischen und souverän-herrischen Blick der ErzählerInnen

1. - Kracht, Christian, Imperium, Köln, Kiepenheuer \& Witsch, 2012, S. 67. Folgend im Text durch die Sigle I in Klammer referenziert; der vorliegende Artikel basiert auf Teilen meiner Publikation: Hauenstein, Robin, Historiographische Metafiktionen, Würzburg, Königshausen \& Neumann, 2014.

2. - Honold, Alexander, ,Literatur in der Globalisierung - Globalisierung in der Literatur", www.germanistik.ch, 2010, S.9. 
in kolonialen Reiseberichten und Kolonialromanen konterkarieren und ablösen wollte ${ }^{3}$.

Trotz der Gemeinsamkeiten fällt daher auf, dass sich Kracht in Imperium einem Erzählmodus, wie er die älteren, postkolonialen Reiseberichte auszeichnet, verwehrt, und stattdessen einen unfokalisierten, olympischen Erzählmodus und -duktus wählt, wie er gerade für den Kolonialroman kennzeichnend ist, und der mitunter für die Diskussion mitverantwortlich ist, die das Erscheinen von Krachts Roman entfacht hat, und im Zuge derer Georg Diez dem Autor und seinem Werk ,,antimodernes, demokratiefeindliches, totalitäres Denken“ und eine ,rassistische Weltsicht“" zugeschrieben hat ${ }^{4}$. Die Naivität, mit der Diez Autor und Erzähler gleichstellt und seine abstruse Engführung rechten Gedankenguts mit Imperium ist erschreckend und erübrigt jeden längeren Kommentar, sie erfuhr damals zudem umgehend Erwiderung durch Felicitas von Lovenbergs Artikel ${ }^{5}$, doch der Aspekt, an dem sie sich entzündet, ist von zentraler Bedeutung für die Position Imperiums und die Bestimmung seines Diskurses. Es ist die Frage, die sich Diez bezeichnenderweise selbst stellt: „Wer spricht da? [...] Wer denkt so?"6.

Der Stil, den sich Kracht in Imperium eindrücklich souverän zu eigen macht, stammt aus der Zeit des deutschen Kaiserreichs selbst. Kracht bedient sich des realistischen Pastiches, dem Duktus Fontanes und Thomas Manns etwa, und setzt einen unfokalisierten Erzähler ein, der die Geschichte mit einer großen Heiterkeit, einer lässigen Distanz, und mit stets ironischem Unterton äußerst detailliert, doch niemals eintönig zu erzählen weiß. Die Allwissenheit des Erzählers wird jedoch zwischendurch, in Zwischenfragen und Bemerkungen zum Eingeständnis des Nichtwissens metafiktional subvertiert: „Govindarajan wies mit einem Stock (hatte er vorhin schon einen dabeigehabt?) Richtung Tempel, [...]“" (I 43). Als Aueckens, bei Kracht Engelhardts erster Jünger, tot aufgefunden wird und die Todesursache unklar bleibt, wie denn auch die historischen Belege keinen Todeshergang kennen, belässt auch Kracht seinen Erzähler im Nichtwissen, die Vorgänge ,verschwinde[n] im Nebel der erzählerischen Unsicherheit“ (I 129).

Diese illusionsstörenden metanarrativen Effekte, die Kracht in die Narration einbaut, sind neben dem Einsatz des ironischen Pastiches

3. - Vgl. dazu grundlegend Lützeler, Paul Michael, „Einleitung”, in: ders. (Hg.), Der postkoloniale Blick. Deutsche Schriftsteller berichten aus der Dritten Welt, Frankfurt am Main, Suhrkamp, 1997, S. 7-33. - Bezüglich des Wilhelminischen Militarismus und der Kolonialpolitik vgl. etwa die Position Friedrich Naumanns: Naumann, Friedrich, Mitteleuropa, Berlin, Reimer, 1915.

4. - Diez, Georg, ,Die Methode Kracht“, in: Der Spiegel, 13.2.2012.

5. - Lovenberg, Felicitas von, „Kein Skandal um Christian Kracht“, in: FAZ, 14.2.2012.

6. - Diez 2012. 
des klassischen Realismus und den ebenfalls unter die metafiktionalen Effekte zu zählenden theoretisierenden Verhandlungen und Verfahren kinematographischer Art auf diskursiv-narratologischer Ebene die postmodernen Kennzeichen des Romans. Aus gattungstheoretischer Sicht kann diese Inszenierung epistemologischer Fragestellungen als ein Aspekt gesehen werden, der den Roman auch als eine fiktionale Metabiographie qualifiziert, deren Themenschwerpunkt auf der Unterminierung biographischer Faktizität liegt und somit einem der drei „Angriffspunkte“, die Nadj für die fiktionale Metabiographie als zentral ausgemacht hat und deren Formen selbstreflexiver Darstellungselemente, auf die auch folgend Bezug genommen sei, sie in metabiographische, metahistorische, metanarrative sowie metafiktionale Referenzen unterteilt ${ }^{7}$.

Das kinematographische Verfahren, das bereits in der Eingangspassage des Romans und ihrer Zoom-Technik zu beobachten ist, wird auch in der Folge immer wieder eingesetzt und explizit metafiktional ausgestellt und damit zur Reflexion gebracht. Seine Krönung findet die Exposition des cineastischen Verfahrens in der finalen, postmodern-ironischen Überführung des Romans in seine eigene Hollywood-Verfilmung, die die eben erzählte Geschichte wieder von vorne beginnen lässt, den Romananfang zum neuerlichen, kommentierten Bild erhebt:

Und tatsächlich wird einige Jahre später vor Publikum, Engelhardt ist nun schon von uns gegangen, getragene, monumentale Musik orchestral aufbranden, der Regisseur ist bei der Premiere in erster Reihe anwesend [...], es rattert der Projektor, nein, es flirren Hunderte Projektoren und werfen ihre von wild tanzenden Staubpartikeln begleiteten Lichtnadeln auf Hunderte Leinwände, in Cincinnati, Los Angeles, Chicago, Miami, San Francisco, Boston, auf denen ein weißes Dampfpostschiff unter langen weißen Wolken durch einen endlosen Ozean begibt. Die Kamera fährt nah heran, ein Tuten, die Schiffsglocke läutet zu Mittag, und ein dunkelhäutiger Statist (der im Film nicht wieder auftaucht) schreitet sanftfüßig und leise das Oberdeck ab, um jene Passagiere mit behutsamem Schulterdruck aufzuwecken, die gleich nach dem üppigen Frühstück wieder eingeschlafen waren. (I 241f.)

Interessant an dieser Passage erscheint auch, dass der ,malaysische Boy“ (I 11) des Romanbeginns hier durch die Bezeichnung „,dunkelhäutiger Statist“ ersetzt wird: Boy war auch in den deutschen Kolonien und

7. - Vgl. Nadj, Julijana, „Formen und Funktionen gattungsspezifischer Selbstreflexivität in der fiktionalen Metabiographie am Beispiel von Carol Shield's Swann", in: Janine Hauthal et al. (Hg.), Metaisierung in Literatur und anderen Medien. Theoretische Grundlagen - Historische Perspektiven - Metagattungen - Funktionen. Berlin, de Gruyter, 2007, S. 327ff. 
im offiziellen kolonialen Diskurs die gebräuchliche Bezeichnung der weißen Kolonialisten für Afrikaner und stärker pigmentierte Personen jeden Alters. Sie entsprang dem sozialdarwinistisch fundierten paternalistischen Standpunkt in der vorherrschenden Sichtweise bezüglich des Umgangs mit den kolonial eroberten Völkern, demzufolge sich die Afrikaner (und die anderen kolonial beherrschten Volksgruppen) noch im entwicklungsgeschichtlichen Stadium der „Kindheit“ befänden ${ }^{8}$. Die zahlreichen dem kolonialen Diskurs zuzuschreibenden rassistischen Versatzstücke des Krachtschen Erzählers finden sich im Roman durchgehend: von den „barbusigen dunkelbraunen Negermädchen“ (I 13) über die Bezeichnung der Halbsamoanerin Emma Forsayth als „Halbblut“ (I 57) bis zu Benennungen der Tolai und ihrer Tätigkeiten als „primitiv“ (I 188). Als Engelhardt erstmals auf Kabakon landet und die Insel für sich in Besitz nimmt, heißt es, die „Eingeborenen“ hätten sich „mit einer gewissen phlegmatischen Neugier am Strand eingefunden“. Dazu hin habe einer, ,als parodiere er sich und seine Rasse, einen Knochensplitter in der Unterlippe“ getragen (I 65f.). Während letztere Aussage schlichtweg ein weiteres explizites rassistisches Versatzstück darstellt, verweist die angebliche „Phlegmatik“ der Bewohner Kabakons hier als implizites Pendant auf die kolonialistische Überzeugung einer angeblichen angeborenen Faulheit der kolonisierten Völker, die, so der Grundsatz der deutschen Kolonialideologie und Kolonialpädagogik, zur Arbeit erzogen werden mussten und sollten ${ }^{9}$. Doch die rassistischen Aussagen finden sich nicht nur in Bezug auf die Melanesier und Mikronesier, auch die Franzosen etwa, als Intimfeinde des Kaiserreichs, werden abgewertet und als „eklatante Snobs“ mit einer ,autistischen Eleganz" bezeichnet (I 135).

Die Beispiele verdeutlichen, dass Krachts Erzähler Versatzstücke des kolonialen Diskurses verwendet, dem auch ein latenter Rassismus inhärent ist. In allen Beispielen handelt es sich jedoch um Fokalisierungen der Perspektive Engelhardts durch den Erzähler. Zudem ist der Erzähler nicht der Autor, und Schwarz hat bereits hervorgehoben, dass es ,zu den Spielregeln des historischen Romans über den Kolonialismus [gehört], dass sich dessen Personal der Sprache der Kolonialherren bedient"10. Dasselbe gilt auch für die antisemitischen Kommentare, wie die Äußerung Aueckens (I 126); sie sind sämtlich der Figurenrede und dem Antisemitismus im Kaiserreich und vorausweisend im Dritten Reich zuzuordnen. Krachts „Methode“ ist folglich nicht die

8. - Vgl. Gründer, Horst, Geschichte der deutschen Kolonien, Paderborn, Schöningh, 2012, S. 237.

9. - Vgl. Gründer 2012, S. 243.

10. - Schwarz, Thomas, „Eine Tragikomödie der Südsee: Marc Buhls und Christian Krachts historische Romane über das imperiale Projekt des August Engelhardt", www.germanistik.ch, 2012, S. 8. 
der Verbreitung rechten Gedankenguts, sondern das ironisch-kritische Zitieren des kolonialen und antisemitischen Diskurses im Rahmen der internen Fokalisierung des ansonsten unfokalisierten Erzählers, der selbst wiederum ein Pastiche des Erzähltons der großen Realisten des 19. Jahrhunderts darstellt.

Trotz dieser Feststellung bleibt die Erzählinstanz Imperiums zweideutig und erfordert eine Leserschaft, die ihre Weltanschauung kritisch mitliest. So etwa, wenn behauptet wird, das 20. Jahrhundert hätte lange Zeit so ausgesehen, als würde darin ,Deutschland seinen rechtmäßigen Ehren- und Vorsitzplatz an der Weltentischrunde einnehmen“ (I 18), oder wenn Hitler als ,deutscher Romantiker“ umschrieben wird (IM 18). Auch die Bezeichnung des homosexuellen Aueckens als "Sodomiten“ (I 131) sowie die Zusammenfassung von „Gaunern, Säufern, Piraten, Methodisten, Strandläufern“ als „unsauberes Charakterwerk“ (I 139) entspringt weniger der Figurenrede als der Rede des heterodiegetischen Erzählers selbst. Von diesem wissen wir nur (an dieser Stelle wird die Diegese gewechselt), dass er wohl nach dem 2. Weltkrieg, wahrscheinlich als Deutscher, geboren sein muss, da zur Zeit des Dritten Reiches seine „Großeltern auf der Hamburger Moorweide“ nicht sehen wollen, wie Leute ,am Dammtorbahnhof in Züge verfrachtet und ostwärts geschickt werden, [...]“ (I 231).

Neben dieser leicht verschlüsselten Bemerkung zum Holocaust findet sich dazu noch eine zweite explizite, an der Stelle, an welcher der Erzähler während der Rückschau auf Engelhardts Zeit in Deutschland bei der Beschreibung von dessen Besuch in München cineastisch zur Feldherrnhalle hinüberschwenkt:

Die Feldherrnhalle, jene florentinische Parodie dort drüben, kaum eines Blickes gewürdigt, steht mahnend, ja beinahe lauernd im spektralen Münchner Sommerlicht. Nur ein paar kurze Jährchen noch, dann wird endlich auch ihre Zeit gekommen sein, eine tragende Rolle im großen Finsternistheater zu spielen. Mit dem indischen Sonnenkreuze eindrücklich beflaggt, wird alsdann ein kleiner Vegetarier, eine absurde schwarze Zahnbürste unter der Nase, die drei, vier Stufen zur Bühne ... ach, warten wir doch einfach ab, bis sie in äolischem Moll düster anhebt, die Todessymphonie der Deutschen. Komödiantisch wäre es wohl anzusehen, wenn da nicht unvorstellbare Grausamkeit folgen würde: Gebeine, Excreta, Rauch. (I 79)

Die Darstellung des Holocausts und der NS-Zeit als „Finsternistheater" und „Todessymphonie“ mag isoliert sicherlich provokant erscheinen, doch die Wortwahl und die hochironische Hitler-Parodie passt stilistisch zur Gesamtheit des tragikomischen Werks, dessen Erzählweise zwar von einer großen Leichtigkeit, aber eben nicht 
Leichtfertigkeit geprägt ist ${ }^{11}$. Denn Kracht geht es gerade auch darum, die Anfänge der späteren Eskalation zu skizzieren und die Geschichte Engelhardts „stellvertretend“ (I 18) für den Weg Deutschlands nachzuzeichnen, was besonders in der durchgehenden Antisemitismus-, Kolonialismus und Wahnsinns-Thematik ersichtlich ist, wie noch genauer $\mathrm{zu}$ illustrieren sein wird. Was Kracht einfordert, ist die Aufmerksamkeit der Lesenden, die den zuweilen subtilen kolonialen und nationalsozialistischen Diskurs im Einsatz des ironischen Erzähler-Zitats auch auf sprachlicher Ebene erkennen, dabei jedoch auch den Erzähler selbst in seiner Weltanschauung und seiner totalitären auktorialen Perspektive kritisch hinterfragen sollen, alleine schon deshalb, weil der souveräne, unfokalisierte Erzählmodus gerade auch derjenige des Kolonialromans ist.

Krachts Handhabung einer postmodernen ironischen Zitierweise unterschiedlicher Diskursformen, am auffallendsten jedoch des kolonialen Diskurses, erzeugt eine Subvertierung derselben, und es stellt sich die Frage, ob diese implizite Kritik auch inhaltlich verhandelt wird. Parallel zu den kuriosen Vorstellungen und dem Vorhaben Engelhardts, der es in seiner Selbstbestimmung als „Prophet" und Missionar“ als seine „Bestimmung“ ansieht, „eine Kolonie der Kokovoren zu erschaffen" (I 20), wird auch die deutsche Kolonie selbst, die Engelhardt zum geeigneten Ort seiner Mission ausersehen hat, von Beginn weg in ihrer Überflüssigkeit und Sinnlosigkeit skizziert, was somit auf den deutschen Imperialismus selbst zurïckgespiegelt wird (I 18). Von den deutschen Bewohnern dieses ,,vollkommen überflüssigen“ (I 18) SüdseeSchutzgebietes zeichnet der Roman zudem kein gutes Bild. So sind denn bereits die „dickleibigen Pflanzer“, die „schmatzend träumten von barbusigen dunkelbraunen Negermädchen" (I 13), mit denen Engelhardt auf der Prinz Waldemar Kurs auf Deutsch-Neuguinea nimmt, von einer „liederliche[n] Erscheinung“ (I 12) und werden in Engelhardts Fokalisierung ironisch lächerlich gestellt: „Bläßliche, borstige, vulgäre, ihrer Erscheinung nach an Erdferkel erinnernde Deutsche lagen dort und erwachten langsam aus ihrem Verdauungsschlaf, Deutsche auf dem Welt-Zenit ihres Einflusses" (I 12). Was sich in Herbertshöhe an Neuankömmlingen einfindet, ist für den Erzähler das „Strand- und Treibgut des Deutschen Kaiserreiches“" (I 30), wobei offensichtlich ist, dass damit auch auf Engelhardt selbst verwiesen wird, der sich freilich vom Rest der Kolonialbevölkerung abgrenzen möchte. Vor allem mit den ,tumben, alkoholkranken Pflanzern“ (I 53), welche die Mehrheit des Deutschen Klubs vor Ort ausmachen, will Engelhardt nichts zu schaffen haben, so dass er dem Klub fernbleibt.

11. - Vgl. von Lovenberg 2012. 
Die theatralische Inszenierung des Imperialismus zum Ziel der Einschüchterung der kolonisierten Völker wird anhand des Vollzugs der Prügelstrafe vor der Gouverneursresidenz evident. Die brutale Peinigung eines „eingeborene[n] Delinquent[en]“ durch die „Schutztruppe“ (I 168) steht in starkem Kontrast zur Erklärung von Gouverneur Hahl, die Bestrafung diene dazu, ,die Eingeborenen in die deutsche, aufrichtig moralische Gesetzbarkeit einzubinden“, die anders als die „modern[e] Form der Sklaverei“ in den belgischen, französischen und niederländischen Kolonien eine „faire Instanz“ und von weit größerer „Menschlichkeit“" sei (I 169). Gleichermaßen verhält es sich später mit Hahls Aussage, er verstehe die Ermordung Engelhardts aufgrund dessen Touchierung seines „Einflußbereich[s]“ durch „Anarchie und Irrsinn“ im Rahmen der „Ausübung gesetzlicher, vernunftvoller Macht“ (I 205). Die ,zynische Ambivalenz von kolonialer Ideologie und Praxis“ wird an diesen Stellen von Kracht kritisch, in der dem Roman inhärenten Tragikomik bloßgelegt ${ }^{12}$.

Mit Engelharts Besitznahme, seiner Ankunft auf Kabakon, beginnt sodann auch dessen persönliches koloniales Projekt, er wähnt sich „einerseits als Herr über das Eiland und somit auch über das Tun und Lassen seiner Einwohner“, möchte ,,andererseits aber auch die Sitten der Eingeborenen dulden“" (I 71). Den Beginn dieser persönlichen Kolonialisierung, den ,entscheidenden Schritt nach vorne auf den Strand“ umschreibt der Erzähler jedoch als ,Schritt zurück in die exquisiteste Barbarei“" (I 67), die mit Engelhardts Ankunft im Herz der Finsternis seines vermeintlichen Südsee-Paradieses anbricht. Der intertextuelle Verweis auf Joseph Conrads Kolonialroman wird dabei bewusst evoziert: Der frühere Besitzer von Engelhardts erworbener Kokosplantage sei, so Emma Forsayth, „wahnsinnig geworden“ und habe „sich, seine Familie und drei schwarze Arbeiter mit Pech übergossen und angezündet", sein Testament könne ferner nicht anerkannt werden, da es nur die Worte Bringt sie alle um enthalte (I 59). Im Bericht von Kurtz in Heart of Darkness heißt es entsprechend: „Exterminate all the brutes!"13. Bezeichnend ist diesbezüglich zudem, dass in der Figur Kurtz', bei Conrad inhaltlich zwar ein kulturelles Hybrid, sprachlich der deutsche Aspekt hervorsticht. Thomas Schwarz hat darauf verwiesen, dass der sogenannte Tropenkoller im literarischen wie öffentlichen Diskurs der Kolonialzeit zu einem spezifisch deutschen, pathologischen Symptom stilisiert wurde (etwa in Frieda von Bühlows Tropenkoller (1896) oder in Henry Wendens Roman gleichen Titels von 1904), was sich in der häufigen deutschen Abstammung der vom zu brutalen Ausfällen

12. - Vgl. dazu grundlegend Schwarz 2012, S. $10 f$.

13. - Conrad, Joseph (1899): Heart of Darkness. London, Penguin, 2000, S. 84; vgl. Schwarz 2012, S. 9. 
führenden Tropenkoller infizierten literarischen Figuren widerzuspiegeln scheint ${ }^{14}$. Auch dieses Element ist bei Krachts Fiktionalisierung Engelhardts sicherlich mitzulesen.

Der Moment der Landnahme und Ankunft Engelhardts wird vom Erzähler zudem in Anlehnung an die „Splitterung der Realität in verschiedene Teile“ (I 66) als Charakteristikum der Epoche perspektivisch gespalten und mit der weiteren metafiktionalen, metahistorischen Bemerkung versehen, der Moment der Besitznahme habe so ganz unterschiedlich ausgesehen, ,je nachdem von welcher Warte man das Szenario betrachtete und wer man tatsächlich war" (I 66):

[F]ür die schwarzen Männer im Boot und die paar Eingeborenen, die sich mit einer gewissen phlegmatischen Neugier am Strand eingefunden hatten, [...] sah es aus, als sei es ein frommer Gottesmann, der dort vor ihnen betete, während es uns Zivilisierte vielleicht an eine Darstellung der Landung des Konquistadoren Hernan Cortes am jungfräulichen Strande von San Juan de Ulua erinnert, allerdings gemalt, falls dies denn möglich wäre, abwechselnd von El Greco und Gauguin, die mit expressivem, schartigem Pinselstrich dem knienden Eroberer Engelhardt die asketischen Züge Jesu Christi verleihen. (I 66)

Die evozierte Parallele zwischen Engelhardt und Cortes, eine der großen europäischen Entdecker- und Eroberer-Figuren, installiert Engelhardt innerhalb einer Traditionslinie der kolonialistischen Expansion. Dies wirkt faktisch gesehen übertrieben, hat jedoch die Funktion, Engelhardt narrativ zum Sinnbild der deutschen Expansion in der Südsee und der nationalen Entwicklung in Richtung Wahn und „Barbarei“ zu stilisieren. Der Verweis auf die mögliche ikonographische Darstellung des sich zum Kolonialherrscher aufschwingenden Engelhardt als Jesus Christus in der Manier der Malerei der Epoche ist insofern doppelt ironisch, als er einerseits die Bildtradition des Abendlandes kritisch unterläuft und andererseits metafiktional auf die Vorgangsweise des Erzählers selbst zurückverweist, der mit bildlichen Überblendungen Engelhardts, auch bezüglich des Heilands, keineswegs spart: Der selbsternannte „Prophet" und „Missionar“ ist der „Protagonist“ der „,neuen Zeit“ (I 23), ein „Führer“ (I 82) und „Erlöser“ (I 61). Letzteres erfährt man durch die Beschreibung Emma Forsayths, die Engelhardt an die Märtyrerdarstellungen bei Fra Angelico erinnert: „Engelhardt war dem Erlöser auf diesen Portraits wie aus dem Gesicht geschnitten“ (I 61).

14. - Vgl. Schwarz, Thomas, „Die Kultivierung des kolonialen Begehrens - ein deutscher Sonderweg?“, in: Alexander Honold und Oliver Simons (Hg.), Kolonialismus als Kultur. Literatur, Medien, Wissenschaft in der deutschen Gründerzeit des Fremden. Tübingen u.a., Francke, 2002, S. 85-102. 
Der Gründer des Sonnenordens wird also auf unterschiedlichen Ebenen ikonographisch und sinnbildlich bestimmt und geformt. Seine Besitznahme Kabakons wird im kolonialistischen Zusammenhang ausgestaltet. „Eine Kolonie der Kokovoren zu erschaffen“ (I 20) ist das Ziel Engelhardts, für das Kabakon nur den Beginn markiert, aus dem sich das fruktivorische Weltreich herausentwickeln soll:

Der Sonnenorden wird zunächst Kabakon besiedeln, von da aus den Bismarck-Archipel, dann Neuguinea und die Inseln des Stillen Ozeans, schließlich das tropische Zentral- und Südamerika, das tropische Asien und das äquatoriale Afrika.

Ich fordere alle Fruktivoren und Freunde der naturgemäßen Lebensweise auf, mitzuhelfen bei dem Bau des Palmentempels des Fruktivorismus, den es aufzurichten gilt, mitzuwirken bei der Gründung des fruktivorischen Weltreichs ${ }^{15}$.

Die Kuriosität des historischen Vorhabens wird von Kracht entsprechend komisch und ironisch gespiegelt. Anstatt als erhofftes Utopia endet der Sonnenorden in „Anarchie und Irrsinn“ (I 205), Mord und Totschlag und sodann mit der völligen Vereinsamung und Zerrüttung Engelhardts, zuletzt in spöttischer Zuspitzung nurmehr eine weitere Touristenattraktion für Südseereisende (I 299). Mit dem Ende des Sonnenordens, pointiert festgehalten im Bild des vollends lächerlich und zur Attraktion gewordenen Wilden am Strand, vollzieht sich auch seine endgültige Destruktion. Was als paradiesisches, philosophisch begründetes Weltreich gedacht war, somit im Diskurs der Kolonialzeit die Zivilisation gegenüber der Barbarei verkörpern sollte, endet in völliger „Archaik“. Dass man den „Propheten“ Engelhardt zuletzt wie „ein wildes Tier im Zoo besucht“" (I 299), wie es die Europäer der Zeit auf dem Kontinent mit den exotischen Tieren aus den Kolonien tun, entspricht dieser Umkehr der Perspektive. So erscheint denn mit der Destruktion des Kolonialisierungsvorhabens Engelhardts, das im Kleinen die deutsche Herrschaft im Pazifik selbst zu reflektieren scheint, auch die Wilhelminische Expansion in den Tropen in ihrer wirtschaftlich, politisch und welthistorisch kaum nennenswerten Bedeutung ironisch subvertiert und implizit metafiktional und kritisch reflektiert.

Der transtextuellen Überblendung der Verdüsterung von Engelhardt und dessen Kolonialprojekt sowie der Destruktion des kolonialen Diskurses mittels seiner eigenen Ordnungsstruktur, die sich in der simplen Binärkodierung von Zivilisation/Kultur und Wildnis/Barbarei

15. - Werbeprospekt August Engelhardts, zit. nach Klein, Dieter, „Neuguinea als deutsches Utopia. August Engelhardt und sein Sonnenorden“, in: Hermann Joseph Hiery (Hg.), Die deutsche Sïdsee. Ein Handbuch. Paderborn, Schöningh, 2001, S. 456. (Im Original kursiv) 
niederschlägt, entspricht zudem, dass Kracht auch den KannibalismusDiskurs ironisch überzeichnet auf Engelhardt selbst überträgt: Der Kannibale gehört seit der Entdeckung der Neuen Welt zu den stabilsten Topoi in den Texten über die außereuropäische Fremde. In diesen Berichten zeigt sich jedoch die Tendenz, die indigenen Völker Australiens sowie die Melanesier als besonders primitiv und minderentwickelt darzustellen, in der Südsee und speziell dem melanesischen Raum den Hauptsitz des Kannibalismus überhaupt auszumachen und die Melanesier in ihrer Kultur und ihrem gesamten Wesen auf die Anthropophagie zu reduzieren ${ }^{16}$. Im Schutzgebiet Deutsch-Neuguinea war Kannibalismus flächendeckend vorhanden, er wurde jedoch zuweilen durch Strafexpeditionen zu unterbinden versucht. Die letzten bezeugten Fälle ereigneten sich um das Jahr 1906. Zu den regionalen Schwerpunkten zählte neben Neumecklenburg auch Neupommern und da besonders die Gazellehalbinsel, worauf sich mit Herbertshöhe, später Rabaul, der Hauptsitz der deutschen Verwaltung befand, der Engelhardts Insel Kabakon östlich vorgelagert war ${ }^{17}$. Kracht zieht es in Imperium jedoch vor, den Kannibalismus auf Melanesien bis auf zwei Ausnahmen, die selbst wiederum die Gestalt von Nacherzählungen haben (I 26, 120f.), unerwähnt zu lassen - und stilisiert stattdessen den Veganer und Kokovoren Engelhardt selbst zum werdenden Kannibalen, wenn er ihn uns erst längere Zeit an seinem Daumen nuckelnd, dann diesen verspeisend zeigt.

Wie die erste stammt auch die zweite Erwähnung vom Erzähler selbst und berichtet von einem Mord an einem Missionar, wenngleich zwanzig Jahre zurückliegend. Dieser „Padre“ (I 120) habe mittels eines Beiles die Idole einer Gruppe von Insulanern des Neulauenburg-Archipels beschädigt und sei dergestalt umgebracht und verspeist worden. Eine dieser beschädigten hölzernen Schnitzfiguren erhält Engelhardt als Geschenk von seinen Arbeitern und stellt sie auf seinen Schrein aus Sandelholz, wo sie in unheilvoller Präsenz die späteren kannibalischen Neigungen Engelhardts heraufzubeschwören scheint. In der Darstellung des Erzählers beginnt Engelhardt sodann nach und nach geistig zu degenerieren, wovon sein einsetzendes Daumenlutschen Zeugnis geben soll (I 133, 144). Die „Marotte“ (I 133) erweist sich jedoch bald auch als erwachende Gier nach Menschenfleisch. Als Kapitän Slütter mit dem Auftrag der Ermordung Engelhardts Kabakon erreicht, wird er sich bald der „Bedrohung“ (I 220) bewusst, die von Makeli und seinem Herrn

16. - Vgl. Haberberger, Simon, „Kannibalismus in Deutsch-Neuguinea“, in: Hermann Joseph Hiery (Hg.) 2001, S. 312-321; Joch, Markus, „Völkerkunde in Neuguinea. Herbst 1888: Otto Finsch rettet die Ehre der Menschenfresser",in: Alexander Honold und Klaus R. Scherpe (Hg.), Mit Deutschland um die Welt. Eine Kulturgeschichte des Fremden in der Kolonialzeit, Stuttgart, Metzler, 2004, S. 127-135.

17. - Vgl. Haberberger 2001, S. 314. 
ausgeht. Die Narration impliziert an dieser Stelle, dass die beiden Slütter im Dschungel in eine Falle locken und umbringen wollen (I 223). Makeli fehlt bereits ein Mittel- und Zeigefinger, wobei wahrscheinlich erscheint, dass er beide an Engelhardt abtreten musste, der seine Kokosdiät aufgegeben hat, wie der metanarrative Erzählerkommentar an der Stelle zeigt (I 218). Engelhardt verspeist dann heimlich genüsslich seinen Daumen und offenbart im Selbstgespräch seine auch philosophisch begründete Mutation vom Kokovoren zum Kannibalen:

Engelhardt sei hier unter unglücklichen Kannibalen, die sich fortentwickelt hätten, weg von ihrem natürlichen, gottgegebenen Instinkt, den ihnen die Missionare mit ihrem Gequatsche ausgeredet hätten, dabei sei alles so denkbar einfach, nicht die Kokosnuß sei die eigentliche Nahrung des Menschen, sondern der Mensch selbst sei es. Der ursprüngliche Mensch des Goldenen Zeitalters ernährte sich von anderen Menschen, ergo der gottgleich Werdende, der nach Elysion Zurückkehrende von sich selbst: God-eater. Devourer of God. Und Engelhardt greift zur Kokosschale, darin er seinen Daumen verwahrt hat, entfernt sorgfältig das Salz von dem abgetrennten Stück und beißt hinein, den Knochen mit den Zähnen zerknackend. (I 221)

Kracht säkularisiert hier den historischen Engelhardt, für den der Kannibalismus moralisch nicht vertretbar ist und der nur den Kokovorismus in den Bereich der Theophagie rückt (,Der Kokosesser ist Gottesser, ist Theophag [...] Der Kokovorismus, die Theophagie, ist der Weg zur vollen Erlösung von Schmerz, Leib und Tod“18). Für den kolonialen Diskurs, dem sich Kracht ironisch annimmt, bedeutet die Wandlung Engelhardts zum Kannibalen und damit nach dieser Logik größtmöglichen Primitivität bei zeitgleicher „Fortentwicklung“ der Insulaner die vollständige Demontage.

Diese inhaltliche Destruktion des Kolonialisierungsvorhabens Engelhardts und damit sinnbildlich auch des deutschen Imperialismus, die kritische Bloßstellung der zynischen Ambivalenz zwischen dessen Herrschaftsideologie und -Praxis sowie Krachts postmodern-ironische Zitatweise des kolonialen Sprachgebrauchs verleiht Imperium durchaus eine postkoloniale Perspektive. Der postkoloniale Blickwinkel in Imperium ist aber, anders etwa als in den zu Beginn erwähnten, früheren Auseinandersetzungen der deutschen Literatur mit der postkolonialen Situation in den ehemals kolonisierten Ländern, nicht auf die nationale Selbstbestimmung, die Findung und Konstituierung einer kulturellen Identität des heutigen Papa-Neuguinea ausgerichtet. Der postkolo-

18. - Engelhardt, August, Das Kokosevangelium, in: Dieter Kiepenkracher (Hg.), Hoch der Äquator! Nieder mit den Polen! Eine sorgenfreie Zukunft im Imperium der Kokosnuss, Norderstedt 2012, S. 105. 
niale historische Roman in Form eines Abenteuerromans, der zudem Tendenzen einer fiktionalen Metabiographie aufweist, wie die metabiographischen und metanarrativen Elemente gezeigt haben, ist vielmehr einer kritischen historiographischen Reflexion zuzuordnen, die mittels der Umkehr binärer Kodierungen, der subtilen Dekonstruktion des kolonialen Diskurses am Beispiel des Projektes Engelhardts operiert. Unerwähnt beziehungsweise außerhalb des Fokus' des Romans bleiben dabei jedoch beispielsweise die brutalen Strafexpeditionen, Scheinprozesse und andere kriegerische Konflikte der Region. Die kritische Reflexion des lange gepflegten Mythos von der idyllischen deutschen Sïdsee wird dergestalt symbolisch und metahistorisch vor allem durch die Dekonstruktion dieses Bildes in der ironisch modellierten Darstellung der Entwicklung Engelhardts und seinem Sonnenorden geleistet.

„Die Geschichte nur eines Deutschen"19 - Vom Wahnsinn der Südsee in die Katastrophe des Dritten Reichs

Die Parallelisierung der Figur Engelhardts mit dem ,späteren deutschen Romantiker und Vegetarier" Hitler (I 18f.), wie sie vom Erzähler schon zu Beginn des Romans vorgestellt wird, macht das Geschehen um den deutschen Aussteiger symbolisch zur Vor-Geschichte der späteren deutschen Katastrophe, mit der es „,in nuce auch kohärent“ (I 19) sei, zu der sich also Verbindungslinien ziehen lassen sollen. Die Geschichte Engelhardts, ausgewählt als Sinnbild der Verwirrung nur eines Deutschen, wird gleichsam „stellvertretend“ (I 18) erzählt für die Geschichten der anderen, die Entwicklung des Nationalstaates hin zum Wahnsinn des Dritten Reichs selbst.

Engelhardt wird bei aller Abscheu seinerseits vor seinen Landsleuten narrativ durchgehend mit deutsch-germanischen Elementen assoziiert und verbunden. Schlafend gleicht er beispielsweise Wagners schlafendem „Jung Siegfried“ (I 36), auf der Fahrt nach Deutsch-Neuguinea träumt Engelhardt auf dem Dampfer noch von einer kultischen Bestattung der Wikinger, von Kindern mit zu Kränzen geflochtenen blonden Haaren (I 27f.). Engelhardt scheint die nordische Symbolik folglich mit in die Südsee zu nehmen, selbst in den fernen Gefilden bricht sie sich im Schlaf in seinen unbewussten Bewusstseinszuständen Bahn und nistet sich dort gewissermaßen ein. Zuvor noch, 1898, Engelhardt befindet sich noch auf seinen Reisen im Kaiserreich, wird er als ebenjener Typus des „deutschen Romantikers“ präsentiert, von dem der Erzähler das spätere Unheil ausgesehen sieht. Engelhardt spaziert im ostpreußischen Memel über den Strand, „,sich fragend, ob vielleicht hier des Deutschen Seele

19. - I 18. 
herstamme, hier, von jenem unendlich melancholischen, einhundert Kilometer langen, sonnenbeschienenen Dünenstrand“ (I 84). Später, die Nacht ist bereits über den baltischen Strand hereingebrochen und Engelhardt starrt, die Südseeträume „langsam in sich reifen lassend wie ein Kindlein“ (I 84), auf die See hinaus, lässt der Erzähler eine ,traurige litauische Melodie“ über der Nehrung verklingen:

Eine traurige litauische Melodie verweht noch über der Nehrung, unnahbar wie die am Firmament blaß blinkenden Sterne und doch unermeßlich vertraut, lieblich und heimelig: Wuchsen einst fünf junge Mädchen schlank und schön am Memelstrand. Sing, sing was geschah? Keines den Brautkranz wand. Keines den Brautkranz wand. (I 85)

Was erst als das deutschtümelnd-romantische Element bekräftigende Strategie erscheint, das leise Verwehen eines einfachen alten Volksliedes, erweist sich auf den zweiten Blick als Anachronismus und unheilvolles Zeichen, das Engelhardt und dessen Gedankenwelt narrativ wiederum an den Verlauf der deutschen Geschichte bindet. Das alte deutsch-litauische Volkslied Zogen einst fünf junge Schwäne wurde erst 1918 weiter bekannt, als es in die Volksliedsammlung Der Liederschrein aufgenommen wurde. Es ist ein Antikriegslied, das sich nach beiden Weltkriegen größerer Verbreitung erfreute, und dazwischen, ab 1935, von den Nazis aus den meisten Liedersammlungen eliminiert wurde. Die von Kracht zitierte Strophe entstammt einer später (1924) notierten Fassung des Liedes, in welchem in früheren Fassungen noch vom Ostsee- statt vom Memelstrand die Rede ist ${ }^{20}$.

Entsprechend der narrativen Verknüpfung Engelhardts mit dem Verlauf der deutschen Geschichte trifft er auf seinen Fahrten durch das deutsche Reich auch auf andere deutsche Protagonisten: Hermann Hesse verpasst er knapp, Thomas und Katja Mann sehen ihn nackt am Memelstrand und sorgen durch eine Anzeige für seine Verhaftung, wobei die Präsenz Katja Manns hierin ein rein fiktiver Griff Krachts ist - wie viele andere geschichtliche Details im Roman, die von Kracht bewusst falsch, aber eben faktisch oft nur knapp ,verschoben“ arrangiert werden ${ }^{21}$, um die Fiktion auch in diesem Bereich subtil metahistoriographisch zu erweitern und die Lesenden durch eine Art geschichtliches Detektivspiel zu involvieren.

20. - Vgl. Boock, Barbara, ,Die Sammlung Patock im Deutschen Volksliedarchiv. Eine kleine Sammlung deutscher Volkslieder 1908 bei Kaschuben gesammelt“, in: Heike Müns (Hg.), Musik und Migration in Ostmitteleuropa, München, Oldenbourg, 2005, S. 319-331.

21. - Vgl. dazu auch Birgfeld, Johannes, „Südseephantasien. Christian Krachts „Imperium“ und sein Beitrag zur Poetik des deutschsprachigen Romans der Gegenwart, in: Wirkendes Wort 62/3 (2012), S. 469f. 
Wenngleich die Erzählinstanz die Geschichte Engelhardts exemplarisch für die seiner Landsleute zu erzählen vorgibt, wird die Figur des Sonnenorden-Gründers doch unverkennbar überhöht und durch die Hitler-Parallelen als unheilvolle deutsche Sonderfigur inszeniert. Er ist der „Erleuchtet[e]“ inmitten eines „Volkes“ von „Untertan[en]“ (I 92), und wird in Vorausdeutung der späteren Geschehnisse auf dem heimischen Kontinent kodiert als im Erlösungswahn befangene Figur, er ist, auch in diesem Diskurs, der „Protagonist“ der anbrechenden neuen Zeit (I 23), ein „Führer“ (I 82) und „Heiland“ (I 61). Sein Weg in die Tropen führt ihn in den Wahnsinn, ,in die exquisiteste Barbarei“ (I 67) und eine ,geistige Archaik“ (I 189), sie endet mit Mord- und Totschlag sowie Kannibalismus. Ein Zustand, der auf dieser Ebene des Romans die große Katastrophe des 20. Jahrhunderts, für den sich der andere vermeintlich „deutsche Romantiker" verantwortlich zeigen wird, vorausbeschwört und versinnbildlicht. So liegt es denn auch nahe, dass der Kolonialist schlussendlich zum Antisemiten wird (I 224).

$\mathrm{Zu}$ Engelhardts Verfall in Wahnsinn und Rassenwahn passt auch der Duktus des erklärten Vorhabens seines realhistorischen Vorbilds, das davon spricht, aus den Mitgliedern des Sonnenordens „große, edle, gute, urgesunde - ganze Menschen [...] züchten“22 zu wollen. Tatsächlich führen die Linien des nationalsozialistischen Rassenwahns mitunter direkt zurück in den Diskurs der Kolonialzeit. Die Panik vor der Hybridisierung speist sich auch aus dem Diskurs über die Tropen als einer „Zone tödlicher Krankheiten und sexueller Exzesse“23. Der bekannte koloniale Tropenhygieniker und Feind der sexuellen Hybridisierung, Claus Schilling, führte später Experimente an Häftlingen im KZ Dachau durch. Der Anthropologe und Eugeniker Eugen Fischer wiederum, später der vielleicht wichtigste Rassentheoretiker der Nationalsozialisten, führte 1908 Untersuchungen an Mischlingen in Deutsch-Südwestafrika durch und leitete daraus rassisch begründete unveränderliche Unterschiede und das Credo einer strikten Rassentrennung $a^{24}$. Das projektierte Kolonialimperium des Dritten Reichs sah eine durch strikte Segregation und „Rassenhygiene“ geprägte koloniale Klassengesellschaft vor. Schwarz hat diesbezüglich betont, der Zusammenhang zwischen kolonialistischer und nationalsozialistischer Rassentheorie sei nicht zu vernachlässigen ${ }^{25}$. Wenngleich „der Weg von Windhoek nach Nürnberg weit, sehr weit" war, was die gesamten Unterschiede in Ideologie, Gesetzgebung, Verfolgungspraxis,

22. - Pamphlet Engelhardts, zit. nach Klein 2001, S. 456.

23. - Vgl. dazu Schwarz, Thomas, ,Die Tropen als Transferzone“, in: Alexander Honold (Hg.), Ost-westliche Kulturtransfers. Postkoloniale Studien in der Germanistik, Bielefeld, Aisthesis, 2011, S. 282.

24. - Vgl. Gründer 2012, S. 247ff.; Schwarz 2011, S. $281 \mathrm{f}$.

25. - Vgl. Schwarz 2002, S. 97. 
Staatsverfassung angeht, wie etwa Birthe Kundrus eingewandt hat ${ }^{26}$, ist die generelle Kohärenz, die der Krachtsche Erzähler herstellen möchte, für diesen Aspekt folglich durchaus nachzuvollziehen. Dasselbe gilt für die Parallele auch im Punkt der Expansionspolitik, wenngleich mit anderer Richtung - das kolonialistische Denken setzte sich im politischen wie im literarischen Diskurs kohärent fort ${ }^{27}$. Auch bei Kracht scheint die NS-Zeit somit trotz der zeitlichen Ansiedlung des Romans im Wilhelminismus wiederum als Hintergrundfolie durch, und das einfache Geschehen wird mit historischen wie historiographischen Diskursen verwoben. Während diese Merkmale für die deutschsprachige historiographische Metafiktion, zu deren Bereich Krachts Werk zu zählen ist, und ihre im Vergleich vielleicht ausgeprägtere geschichtliche Reflexivität besonders charakteristisch scheinen, wartet Imperium dagegen auch mit literaturhistorischen Spielereien wie auch einer schlichtweg runden und funktionierenden Story auf 28 .

Der historische Engelhardt starb 1919, Kracht lässt ihn jedoch leben bis nach dem Ende des Zweiten Weltkrieges, was die Repräsentationsfunktion Engelhardts als Symptom und Beispiel für den Verlauf der deutschen Geschichte nochmals stark unterstreicht. Als ihn amerikanische Marineeinheiten auf der durch „Kämpfe verwüsteten“ Solomoneninsel Kolombangara in der Erdhöhle eines rauchenden Vulkans entdecken, ist seine Lepra, wie ein synekdochisches Symptom der katastrophalen Entwicklung Deutschlands ,wie durch ein Wunder völlig verheilt“ (I 239). Auf der Militärbasis bekommt der frühere Gesundheitsapostel und Kokovore Coca Cola und Hot Dogs serviert und erlebt so in der ironischen Manier des Werks noch die Befreiung und Umerziehung mit: ,dies ist nun das Imperium“ (I 240) heißt es da, es hat das alte deutsche „Imperium“ (I 231), von dem zehn Seiten zuvor noch die Rede ist und von dessen Ausmaß Engelhardt einst selbst geträumt hat, gestürzt und besiegt. Die ironisch-kritische Skizzierung auch des neuen Imperiums wie der Invasion der deutschen Kolonie durch australische Einheiten zu Ende des Ersten Weltkrieges mahnt jedoch daran, dass hinter jeder neuen Machtausdehnung stets auch schon die brutale Schattenseite lauert:

26. - Vgl. dazu Kundrus, Birthe, „Von Windhoek nach Nürnberg? Koloniale „Mischehenverbote" und die nationalsozialistische Rassengesetzgebung“, in: Birthe Kundrus (Hg.), Phantasiereiche. Zur Kulturgeschichte des deutschen Kolonialismus, Frankfurt am Main, Campus, 2003, S. 110-131. Zitat S. 126.

27. - Vgl. Lützeler, Paul Michael, Postmoderne und postkoloniale deutschsprachige Literatur. Diskurs - Analyse - Kritik. Bielefeld, Aisthesis, 2005, S. 97.

28. - Vgl. zu dieser Gesamtthematik weiterführend Hauenstein 2014; Birgfeld 2012 sieht Imperium ebenfalls als historiographische Metafiktion, die zugleich ernst (Verhandlung von Wahrheitskonstruktionen) und ironisch-unterhaltend agiert; vgl. daher auch Birgfeld 2012, S. 475ff. 
[E]in Paradiesvogel, der sich, vom Lärm aus dem Urwald getrieben, nach Rabaul hineinverwirrt, wird lebendigen Leibes seiner Federn beraubt, Soldaten stecken sich die am Kielende noch blutigen Daunen an ihre Südwester, der nackte, vor Schmerz kreischende Vogel wird, nachdem man ihn auf den Namen Kaiser Wilhelm getauft hat, unter prustendem Gelächter wie ein Rugbyall hin und her gekickt; [...] (I 233)

So bleibt am Ende fast zu vermuten, die metafiktionale Transformation des Textes in den beschriebenen Film, der Krachts Roman beschließt und zugleich das Geschehen von neuem aufzurollen beginnt, sei eine pessimistische Reflexion über die ständige Wiederkehr des Gleichen in der Geschichte.

\section{Bibliographie}

Birgfeld, Johannes, „Südseephantasien. Christian Krachts „Imperium“ und sein Beitrag zur Poetik des deutschsprachigen Romans der Gegenwart, in: Wirkendes Wort 62/3 (2012), S. 457-477.

Boock, Barbara, „Die Sammlung Patock im Deutschen Volksliedarchiv. Eine kleine Sammlung deutscher Volkslieder 1908 bei Kaschuben gesammelt", in: Heike Müns (Hg.), Musik und Migration in Ostmitteleuropa, München, Oldenbourg, 2005, S. 319-331.

Conrad, Joseph (1899): Heart of Darkness. London, Penguin, 2000.

Diez, Georg, „Die Methode Kracht“, in: Der Spiegel, 13.2.2012.

Gründer, Horst, Geschichte der deutschen Kolonien, Paderborn, Schöningh, 2012.

Haberberger, Simon, „Kannibalismus in Deutsch-Neuguinea“, in: Hiery (Hg.) 2001, S. 312-321.

Hauenstein, Robin, Historiographische Metafiktionen, Würzburg, Königshausen \& Neumann, 2014.

Hiery, Hermann Joseph (Hg.), Die deutsche Südsee. Ein Handbuch. Paderborn, Schöningh, 2001.

Honold, Alexander, „Literatur in der Globalisierung - Globalisierung in der Literatur", www.germanistik.ch, 2010.

Joch, Markus, ,,Völkerkunde in Neuguinea. Herbst 1888: Otto Finsch rettet die Ehre der Men-schenfresser", in: Alexander Honold und Klaus R. Scherpe (Hg.), Mit Deutschland um die Welt. Eine Kulturgeschichte des Fremden in der Kolonialzeit, Stuttgart, Metzler, 2004, S. 127-135.

Klein, Dieter, „Neuguinea als deutsches Utopia. August Engelhardt und sein Sonnenorden“, in: Hiery (Hg.) 2001, S. 450-458.

Kracht, Christian, Imperium, Köln, Kiepenheuer \& Witsch, 2012. 
Kundrus, Birthe, ,,Von Windhoek nach Nürnberg? Koloniale „Mischehenverbote“ und die nationalsozialistische Rassengesetzgebung“, in: dies. (Hg.), Phantasiereiche. Zur Kulturgeschichte des deutschen Kolonialismus, Frankfurt am Main, Campus, 2003, S. 110-131.

Lovenberg, Felicitas von, „Kein Skandal um Christian Kracht“, in: FAZ, 14.2.2012.

Lützeler, Paul Michael, „Einleitung”, in: ders. (Hg.), Der postkoloniale Blick. Deutsche Schriftsteller berichten aus der Dritten Welt, Frankfurt am Main, Suhrkamp, 1997, S. 7-33.

Lützeler, Paul Michael, Postmoderne und postkoloniale deutschsprachige Literatur. Diskurs - Analyse - Kritik. Bielefeld, Aisthesis, 2005.

Nadj, Julijana, ,Formen und Funktionen gattungsspezifischer Selbstreflexivität in der fiktionalen Metabiographie am Beispiel von Carol Shield's Swann", in: Janine Hauthal et al. (Hg.), Metaisierung in Literatur und anderen Medien. Theoretische Grundlagen - Historische Perspektiven - Metagattungen Funktionen. Berlin, de Gruyter, 2007, S. 321-339.

Schwarz, Thomas, ,Die Kultivierung des kolonialen Begehrens - ein deutscher Sonderweg?", in: Alexander Honold und Oliver Simons (Hg.), Kolonialismus als Kultur. Literatur, Medien, Wissenschaft in der deutschen Gründerzeit des Fremden. Tübingen u.a., Francke, 2002, S. 85-102.

Schwarz, Thomas, ,Die Tropen als Transferzone“, in: Alexander Honold (Hg.), Ost-westliche Kulturtransfers. Postkoloniale Studien in der Germanistik, Bielefeld, Aisthesis, 2011, S. 269-291.

Schwarz, Thomas, „Eine Tragikomödie der Südsee: Marc Buhls und Christian Krachts historische Romane über das imperiale Projekt des August Engelhardt", www.germanistik.ch, 2012. 
